EDUCACIÓN PARA EL CONSUMO: APROXIMACIÓN EMPÍRICA A LOS HÁBITOS DE CONSUMO DEL ALUMNADO DE SECUNDARIA

EDUCATION FOR CONSUMPTION: EMPIRICAL APPROXIMATION TO THE CONSUMPTION HABITS OF HIGH SCHOOL STUDENTS

\author{
Volumen 15, Número 1 \\ Enero - Abril \\ pp. 1-24
}

Este número se publicó el $1^{\circ}$ de enero de 2015

DOI: dx.doi.org/10.15517/aie.v15i1.16969

Llarela Berríos Valenzuela

María Rosa Buxarrais Estrada

Revista indizada en REDALYC, SCIELO

Revista distribuida en las bases de datos:

CATÁLOGO DE LATINDEX, IRESIE, CLASE, DIALNET, DOAJ, E-REVIST@S, SHERPA/ROMEO, QUALIS, MIAR

Revista registrada en los directorios:

ULRICH'S, REDIE, RINACE, OEI, MAESTROTECA, PREAL, CLACSO 


\title{
EDUCACIÓN PARA EL CONSUMO: APROXIMACIÓN EMPÍRICA A LOS HÁBITOS DE CONSUMO DEL ALUMNADO DE SECUNDARIA EDUCATION FOR CONSUMPTION: EMPIRICAL APPROXIMATION TO THE CONSUMPTION HABITS OF HIGH SCHOOL STUDENTS
}

\author{
Llarela Berríos Valenzuela \\ María Rosa Buxarrais Estrada²
}

\begin{abstract}
Resumen: Este artículo presenta los resultados de la investigación sobre los hábitos de consumo de los estudiantes de secundaria de la provincia de Barcelona, España. La investigación se sitúa en el paradigma cuantitativo, utilizando la metodología ex post facto descriptiva por encuesta, empleando un cuestionario como instrumento de recolección de datos. Debido al carácter diagnóstico y exploratorio, participaron de la muestra 232 estudiantes de 14-16 años, de institutos públicos de las comarcas del Maresme, Vallés Occidental y Barcelonés. Los principales resultados indican que los adolescentes tienen hábitos de consumo moderados, ya que no se consideran consumistas, perciben que influyen medianamente en el consumo familiar y aseguran que han recibido suficiente educación para el consumo responsable, pero lo interesante es que indican que les gustaría aprender más sobre este tipo de consumo. Sin embargo, señalan no tener conocimiento de organismos o entidades a los que se pueden dirigir en caso de reclamación. Se concluye que es necesario abordar la educación para el consumo a través de una propuesta pedagógica desde el ámbito de los valores, y es fundamental trabajar con el trinomio esencial de la educación (familia, escuela, adolescentes), para que contribuya de forma integral en la formación del alumnado de secundaria, a través del desarrollo de actitudes críticas y reflexivas frente a la sociedad de consumo en la que viven y, de este modo, estar preparados como ciudadanía activa y responsable, ante las problemáticas que nos afectan en el siglo XXI.
\end{abstract}

Palabras clave: ADOLESCENTES, CONSUMO, HÁBITOS, EDUCACIÓN SECUNDARIA, EDUCACIÓN PARA EL CONSUMO, ESPAÑA

\begin{abstract}
In this article, the results of the investigation, whose objective was to understand the consumption habits of teenagers in the province of Barcelona, Spain, are described. Due to the diagnostic and exploratory character of the investigation, 232 students from diverse schools participated in the sample. The investigation is situated in a qualitative paradigm, utilizing an ex post facto research methodology of collecting descriptions through polls; the instrument of data recollection is a questionnaire with close-ended questions. The main results indicate that teenagers exhibit some moderate consumption habits, since they do not consider themselves consumerists. The teenagers perceive that these habits moderately influence the overall family consumption, ensuring that they have received enough education to understand consumption. However, it is necessary to embark on an education for consumption through a pedagogical proposition that would help teenagers in becoming critical thinkers when facing the society of consumption in which they live, and, in that manner, be prepared to be responsible citizens when facing the problems that we are exposed to in the XXI Century.
\end{abstract}

Key words: TEENAGERS, CONSUMPTION, HABITS, HIGH SCHOOL EDUCATION, CONSUMER EDUCATION, SPAIN

\footnotetext{
${ }^{1}$ Profesora investigadora Universidad de los Andes, Chile. Doctora en Pedagogía por la Universitat de Barcelona. Dirección electrónica: Ilarela.berrios@uandes.cl

${ }^{2}$ Catedrática Universitat de Barcelona, Departamento de Teoría e Historia de la Educación, España. Dirección electrónica: mrbuxarrais@ub.edu
}

Artículo recibido: 13 de junio, 2014

Enviado a corrección:7 de julio, 2014

Aprobado: 20 de octubre, 2014 


\section{Introducción}

Aproximarse a la compleja realidad de los hábitos de consumo y la educación para el consumo en el alumnado de secundaria, no es tarea fácil, ya que se debe considerar que los hábitos se encuentran condicionados principalmente por el factor social. Y en este ámbito, nos topamos con la familia como la unidad fundamental, la escuela, las relaciones interpersonales y la sociedad de consumo.

La sociedad está inmersa en un periodo caracterizado por un ritmo de cambio social acelerado, producto de una "sociedad de mercado" que nos condujo a una "sociedad de consumo", cuyo concepto ha sido objeto de reflexión de varios intelectuales. Autores como Álvarez y Álvarez (1988), señalan que la sociedad de consumo es "la sociedad de la abundancia, la masificación, la homogeneidad".

Se considera que la "sociedad de consumo" tiene un efecto incuestionablemente deshumanizador, que se podría ejemplificar con el "tener frente al ser", formulado por Fromm (1976). Hoy, el consumo es utilizado como símbolo de estatus, generador de una apariencia y la consiguiente búsqueda del prestigio social. En la adolescencia, los jóvenes se encuentran en el proceso de la búsqueda de identidad y se sitúan en los nuevos roles sociales que deben desempeñar. Uno de ellos es el de "consumidor" y, aunque desde pequeños han sido consumidores, es en esta etapa de la vida cuando pueden tomar conciencia de lo que esto significa (Carosio, 2008; Ersoy-Quadir, 2012; Isaksen y Roper, 2008; Shim, Serido y Barber, 2011). Sin embargo, muchos no cuentan con los conocimientos necesarios para ejercer un consumo responsable.

El artículo presenta una investigación llevada a cabo en la provincia de Barcelona, España. El objetivo general consistió en: "conocer los hábitos de consumo del alumnado de secundaria". Y se organiza de la siguiente manera: Referente teórico, en el cual se abordan los constructos teóricos (adolescentes y consumo, educación para el consumo en los adolescentes). Metodología, en este apartado se sitúa el tipo de metodología empleada, la muestra, el instrumento y el análisis de datos. Resultados, se exponen de forma descriptiva a través de tablas. $Y$ finalmente, se presenta la discusión y las conclusiones de la investigación. 


\section{Referente teórico}

\subsection{Adolescentes y consumo}

La incorporación de los adolescentes dentro del círculo del consumo no es nada nuevo. En realidad, esta inserción se produce en la infancia, cuando los padres, las madres y la familia en general, actúan como mediadores del consumo (Chaplin y John, 2010; Flouri, 1999).

La adolescencia de la sociedad occidental, principalmente de países desarrollados y en vías de desarrollo, percibe dinero con frecuencia e incrementa su poder adquisitivo día a día, aunque como consumidores este aún es limitado (Osuna, 2008). Sus acciones de consumo están vigiladas por sus familias y no tienen una gran capacidad de ahorro, por lo que el espectro de productos a los que pueden acceder por cuenta propia es limitado, y sus compras son escasas, según Fernández Cavía (2000).

Los adolescentes de 12 a 19 años se han convertido en grandes consumidores. Generan un mercado sólido para todas las empresas, principalmente porque disponen de una mayor cantidad de dinero con el que pueden tomar sus propias decisiones de compra, así como ejercer sus deberes y derechos como consumidores e influenciar en el consumo familiar (Flouri, 2004; Shim et al., 2011).

Estos cambios son importantes para el rol que los adolescentes interpreten como consumidores. Se debe tomar conciencia de que supone un periodo fundamental en su educación como consumidores (Shavitt, Torelli y Wong, 2009; Sweeting, Hunt, y Bhaskar, 2012). Es imprescindible señalar que en esta etapa se consolida definitivamente el sistema de valores personales (con posterioridad se pueden experimentar cambios ${ }^{3}$ ), por lo que muchos de los hábitos de consumo que adquieren en ella, los mantendrán a lo largo de toda su vida (Chaplin y Lowrey, 2010).

En esta línea, la comercialización de productos respecto al estudio del consumidor adolescente será de mayor importancia. Primero, porque se los mira como clientes potenciales en el futuro. Investigaciones como Outlook Teens 2010, apuntan hacia cómo evolucionarán sus hábitos de consumo en los próximos años, con el objetivo de entender sus valores y anticiparse a sus futuras necesidades. Se trata de una estrategia para captar clientes lo antes posible. El resultado apunta a la posibilidad de que la adolescencia escoja

\footnotetext{
3 Estos cambios, excepcionalmente pueden llegar a ser profundos. Es lo que los sociólogos denominan resocialización: un proceso en el cual la persona asimila un sistema de valores diferentes al que había tenido antes. Este se genera solo en situaciones extremas.
} 
ciertas marcas a las que permanecer fieles durante su vida adulta. Según Kapferer (1992), las marcas escogidas entre los 7 y 18 años continúan siendo las elegidas hasta pasados los 20 años y más.

Los jóvenes están inmersos en un contexto social a través del cual el disfrute de la vida, la búsqueda de la identidad y la felicidad, se han convertido en ejes centrales del proyecto de vida y el consumo actúa como nexo esencial para su consecución; según García Canclini, (1995, p. 14):

(...) las luchas generacionales acerca de lo necesario y lo deseable muestran otro modo de establecer las identidades y construir lo que nos distingue. Nos vamos alejando de la época en que las identidades se definían por esencias ahistóricas: ahora se configuran más bien en el consumo, dependen de lo que uno posee o es capaz de llegar a apropiarse $(\ldots)$

Es entonces cuando el consumismo se convierte en un valor social relevante (Posadas, 2013), que condiciona las aspiraciones de los adolescentes y sus comportamientos.

Existe un modelo diferencial de consumo entre los jóvenes. Para ellos, el consumo satisface necesidades específicas y cumple funciones distintas a las de los adultos. En este sentido, es necesario tener en cuenta algunos elementos primordiales que caracterizan los hábitos de consumo más frecuentes en la juventud. Éstos han sido destacados, tanto desde la investigación sociológica como desde los estudios de mercado. Según Sánchez, Megías y Rodríguez (2004), existen diversos tipos de jóvenes de acuerdo con las variables: sexo, edad, nivel educativo, nivel social, entre otros. Por otro lado, es importante considerar que los hábitos de consumo de adolescentes difieren según la edad, el sexo, y las diferentes etapas de la evolución y maduración personal. Se puede ejemplificar esto indicando que la variables edad y sexo constituyen una gran diferencia en la segmentación del mercado y el consumo, debido a que las estrategias de marketing difieren en función de a quién van dirigidas: varones o mujeres, adolescentes de 13 a 15 años, o de 17 a 20, ya que varían los productos que consumen, la capacidad de compra, las necesidades y los mecanismos de comunicación, entre otros.

Autores como Martín y Velarde (2000) señalan que es posible establecer dos modalidades primordiales del consumo juvenil (consumo e identidad y consumo e 
incorporación a la vida adulta), con base en la función que cumple en las distintas etapas, en el tránsito de la juventud hacia la adultez.

El consumo está asociado a los procesos de identificación juvenil, se utiliza para fortalecer esa identidad y, a su vez, sirve para diferenciarse de los otros (pares y adultos). A este aspecto pertenecen todos los gastos que realiza la juventud para ser aceptada y participar en las relaciones que establece con los demás. De acuerdo con Autio (2002, p.338): “actualmente se describe a la juventud de forma que hace que la generación del 'yo' parezca una generación de activistas filántropos y sociales. Se dice que solo piensan en ellos y que están obsesionados por la gratificación material a corto plazo".

Durante el proceso de identificación, el grupo de pares tiene un papel importante, ya que buscan su aceptación e integración, lo que se logra consumiendo unos productos determinados. Este tipo de consumo para la identificación juvenil tiene como característica principal, centrarse en los espacios que la juventud destina al ocio de carácter social, en los que se comparte los fines de semana y en ambientes públicos.

\subsection{La educación para el consumo en los adolescentes}

La educación para el consumo ha tenido una amplia incidencia en las formas de consumo. En los últimos años se ha producido un gran debate sobre los cambios educativos como forma de hacer frente a los problemas que se plantean en este siglo, entregando herramientas a hombres y mujeres que les permitan desenvolverse dentro de esta sociedad como personas autónomas, responsables y críticas ante las problemáticas sociales (Goldsmith y Clark, 2012; Hill y Beatty, 2011; Isaksen y Roper, 2008).

Para contrarrestar el efecto del consumismo en nuestros jóvenes, es preciso que el consumidor tome conciencia de sus decisiones, tanto en la valoración de sus necesidades como en la influencia que ejercen el mercado y la sociedad en él (Cohen, y Bernard, 2013; Griskevicius y Kenrick, 2013).

En este sentido, la educación del consumidor adquiere una importancia considerable, ya que los jóvenes de hoy quieren tener un buen nivel de vida, lo que no implica calidad de vida. Según el Ministerio de Sanidad y Consumo de España (2004), para abordar estos aspectos debemos diferenciar bien los términos: primero, el nivel de vida, que se puede definir como la expresión de la acumulación y posesión de bienes y recursos; segundo, la calidad de vida, que consiste en el uso y disfrute de bienes y recursos, encaminado al logro del bienestar y la salud. 
La educación para el consumo no ha sido tratada hasta el momento con la atención y la importancia social que merece. En nuestra sociedad de consumo, la familia tiene un papel central como constructora de la identidad personal y del entorno social (Baker, Moschis, Benmoyal-Bouzaglo y Pizzutti, 2013). Los adolescentes tienen a su alrededor una enorme cantidad de bienes, servicios y marcas que invaden su vida cotidiana, y que los puede llevar a convertirse en consumistas (Flouri, 2004; Roberts, Manolis y Tanner, 2008). En este aspecto, la familia tiene un rol esencial como fuente de aprendizaje de hábitos de consumo responsable (Wang, Holloway, Beatty y Hill, 2007).

Hay que ser realista y no obviar que la adolescencia ocupa un lugar destacado en el consumo familiar. La influencia que ejercen los adolescentes en las compras de sus padres y madres, es un aspecto relevante desde hace tiempo en diversos ámbitos, principalmente, el marketing (Belch, Krentler y Willis-Flurry, 2005; Crane, 2010; Park y John, 2011). Los adolescentes son atractivos no sóolo por su capacidad de consumo y ahorro, sino también por su incidencia en el consumo de sus grupos familiares (Roberts, Manolis y Tanner, 2006; Sener, 2011). Respecto a temas de ocio, alimentación y servicios educativos, los adolescentes son un interlocutor del mercado (Lazo y Gabelas, 2007; Shim et al., 2011). Por éste y otros motivos, la adolescencia es una fase considerada clave para la socialización del consumidor y se debe abordar su educación, ya que es en esta etapa cuando se experimentan los primeros contactos directos, autónomos y reales, con el fenómeno del consumo. En estas aproximaciones iniciales los adolescentes se sienten solos, desorientados, buscan ayuda, apoyo, consejos en sus amistades y, ocasionalmente, recurren a sus padres. Esto obedece principalmente a que la sociedad no tiene conciencia de enseñar a ser consumidor y debe facilitar a sus miembros un aprendizaje completo al respecto (Cantera, 2002).

Así pues, la educación para el consumo debe ser asumida por las familias, la escuela, la sociedad y el mercado, por su capacidad transversal, y constituir una educación para la vida (Saldaña y Ballesteros, 2011). Se insiste en que la educación para el consumo no ha sido tratada con la atención y la importancia social que merece. De acuerdo con Benn (2002, p. 169): "en lo referente al tema de la investigación del consumidor se debería poner énfasis en arrojar luz sobre los diferentes aspectos de la conducta del consumidor que se manifiestan a nivel individual, de mercado y social." 
La educación para el consumo será necesaria como estímulo de la evolución con unas actitudes más activas y conscientes. Como afirman Álvarez y Álvarez (1988, p.160) (Sic):

(...) la educación del consumidor/a ha de concebirse como un proceso permanente, que tiene por finalidad aportar al consumidor/a, de forma gradual, secuenciada y acorde con su nivel evolutivo, toda una serie de elementos cognitivos, procedimientos mentales y técnicas de trabajo que le permitan desarrollar actitudes conscientes, críticas y solidarias, sintiéndose satisfecho de actuar de forma responsable ante los hechos de consumo $(. .$.

Desde la perspectiva de Álvarez y Álvarez (1988) y Buxarrais (2003), se considera que la educación del consumidor debe basarse en una educación permanente y pertinente con el desarrollo de la persona, otorgando las herramientas adecuadas que le permitan tomar una postura crítica y responsable ante el consumo. Pujol (1996) indica que en 1976, la Organización Europea de Consumidores (BEUC) realizó un estudio, Education du consommateur dans les escoles, en el cual se observan cuatro posiciones distintas en relación con qué se entiende por educación del consumidor, a nuestro parecer aún vigentes: liberal, reformista, responsable y radical. De acuerdo con el propósito de la presente investigación, nos adentraremos en el concepto de la posición responsable descrita por Pujol (1996, p. 36):

(...) desde esta situación, el consumidor/a debe, colectivamente, revindicar todas aquellas medidas legislativas que regulen los actos de producción. En este marco la educación del consumidor/a, supone dotar a éstos de elementos que les responsabilicen en las consecuencias que sus actuaciones tienen a nivel social y a nivel del medio ambiente; (...) educar al consumidor comporta, desde esta postura, además de todo lo dicho en las posiciones anteriores, dotarle de elementos para que pueda analizar y valorar los efectos sociales y ambientales que se producen en el proceso de producción, comercialización y consumo (...) y actuar consecuentemente.

La descripción de esta posición sobre la educación para el consumo, aporta una valiosa información para abordar la temática, en tanto enfatiza aspectos que, desde la presente perspectiva, constituyen un todo, y son ineludibles para la formación integral de la persona. 
Al mismo tiempo, la educación para el consumo debe posibilitar distinguir entre las necesidades reales y las inducidas, cuestionando el consumo irracional y fomentando el sentido necesario para incidir en las instancias donde se toman decisiones que afectan a los consumidores (Cortina, 2002).

La educación para el consumo propone lograr la transformación del consumidor (niño, jóvenes, adultos) de un agente pasivo en una persona activa, crítica consigo misma, con sus necesidades, y también con la sociedad y los mecanismos que regulan las relaciones de consumo. Por lo tanto, desde una perspectiva pedagógica, es absolutamente necesario incidir desde la adolescencia en la educación del consumidor (Arnould y Thompson, 2005; Burroughs y Rindfleisch, 2002; Pham, 2013).

\section{Metodología}

La investigación se sitúa en el paradigma empírico analítico, también denominado cuantitativo, y se utilizó el tipo de estudio ex post facto descriptivo por encuesta. Esta modalidad investigadora analiza los fenómenos educativos relevantes y las variables que intervienen; fue seleccionada porque responde al objetivo general: "conocer los hábitos de consumo del alumnado de secundaria”.

El diseño de la investigación se basa en los criterios de la metodología de tipo cuantitativa ex post facto, con su aplicación en cuatro fases, según se muestra en la tabla 1.

Tabla 1. Fases de la investigación

\begin{tabular}{|ll|}
\hline \multicolumn{2}{|l|}{ Fases } \\
\hline 1. & Descripción de la situación objeto de estudio \\
\hline 2. & Análisis de datos \\
\hline 3. & Interpretación del significado de los datos \\
\hline 4. & Elaboración de las conclusiones \\
\hline
\end{tabular}

\subsection{Muestra}

Según Hernández, Fernández y Baptista (2003, p. 302), en el enfoque cuantitativo "la muestra es una selección de un subgrupo de la población del cual se recolectan los datos, y que se define o delimita de antemano con precisión". En esta ocasión se optó por un muestreo de tipo intencional. La selección de la muestra del alumnado se realizó bajo los criterios que se muestran en la tabla 2: 
Tabla 2. Criterios de la muestra

\begin{tabular}{|ll|}
\hline 1. & Edad: 14-16 años. \\
\hline 2. & Sexo: femenino / masculino. \\
\hline 3. Institutos de educación secundaria (IES) de carácter \\
público, de la provincia de Barcelona.
\end{tabular}

Fuente: elaboración propia

La muestra corresponde a $\mathrm{N}=232$ (el 59,1\%, varones, y el 40,9\%, mujeres), entre 1416 años, con una media de 15 . Se eligió esta franja de edad porque consideramos que es en esta etapa, donde se construyen los modelos sociales, culturales y la comprensión de la realidad que nos rodea, y es una fase determinante para la vida adulta.

Debido al carácter exploratorio de la investigación en la provincia de Barcelona, se optó por encuestar en institutos de diferentes zonas de la provincia; por este motivo, el IES Sector Sagrada Familia, pertenece a la comarca del Barcelonés; el IES Sector Canet de Mar, a la comarca del Maresme, y el IES Sector Tarrassa, a la comarca del Vallés Occidental. La selección de establecimientos de carácter público, obedece principalmente a que necesitábamos conocer los hábitos de consumo del alumnado de secundaria de este segmento socioeconómico, con la finalidad de plantear líneas de acción pedagógicas en esta área y para este segmento en concreto. La muestra definitiva quedó constituida tal y como se muestra en la tabla 3.

Tabla 3. Muestra definitiva

\begin{tabular}{|l|c|c|}
\hline \multicolumn{1}{|c|}{ Instituto } & Alumnos & $\%$ \\
\hline IES Sector Sagrada Familia & 84 & 36,2 \\
\hline IES Sector Canet de Mar & 78 & 33,6 \\
\hline IES Sector Tarrassa & 70 & 30,2 \\
\hline Total & 232 & 100 \\
\hline
\end{tabular}

Fuente: elaboración propia

\subsection{Instrumento de recolección de la información}

Se elaboró un cuestionario con 20 preguntas cerradas, a partir de la dimensión teórica de consumo. Para asegurar la validez del instrumento, éste fue sometido a la prueba de consistencia interna del coeficiente Alpha de Cronbach, el cual se basa en el promedio de las correlaciones entre los ítems. Arrojó una fiabilidad $\alpha=0,73$, considerada satisfactoria. Las preguntas del cuestionario son de dos tipos: cerradas de tipo excluyente y cerradas de tipo ordinal por intensidad, con las que se trabajó con cinco categorías que aluden a la escala de Likert: 1 = Nunca; 2 = Un poco; 3 = Habitualmente; 4 = Bastante; 5 = Mucho. Para graficar el proceso de elaboración del cuestionario, se adjunta la tabla de especificación empleada al construirlo: subdimensión (aspecto específico del constructo teórico de consumo, en este 
caso de actitudes ante el consumo), los indicadores (se establecen de la profundización y la especificación de la subdimensión) y las preguntas (las cuales constituyen el aspecto de operatividad del proceso de construcción del instrumento) esto queda reflejado en la tabla 4.

\begin{tabular}{|c|c|c|}
\hline SUBDIMENSIÓN & INDICADORES & PREGUNTAS \\
\hline \multirow{6}{*}{$\begin{array}{l}\text { Actitudes ante el } \\
\text { consumo }\end{array}$} & > Principal afición & $\begin{array}{l}\text { ¿Cuál es tu principal afición o hobby? } \\
\text { *Pregunta cerrada de tipo excluyente }\end{array}$ \\
\hline & \multirow{4}{*}{$\begin{array}{l}>\text { Hábitos de } \\
\text { compra }\end{array}$} & $\begin{array}{l}\text { ¿En qué te fijas a la hora de comprar un producto? } \\
\text { ¿Aspectos que consideras para comprar un producto? } \\
\text { ¿Motivos en la decisión final de compra? } \\
\text { ¿Pregunta cerrada de tipo ordinal por intensidad }\end{array}$ \\
\hline & & $\begin{array}{l}\text { ¿Gasto de dinero semanal? } \\
\text { ¿En qué gastas principalmente tu dinero? } \\
\text { ¿Frecuencia de compra mensual? } \\
\text { ¿Quién te acompaña en las compras? } \\
\text { ¿Quién te gusta que te acompañe en las compras? } \\
\text { ¿Preguntas cerradas de tipo excluyente }\end{array}$ \\
\hline & & $\begin{array}{l}\text { ¿Por qué te gusta que te acompañe esa persona? } \\
\text { *Pregunta cerrada de tipo ordinal por intensidad }\end{array}$ \\
\hline & & $\begin{array}{l}\text { ¿Quién decide lo que compras? } \\
\text { ¿Te consideras consumista? } \\
\text { ¿Te agrada comprar productos de marca? } \\
\text { ¿Cómo consideras que son los productos de marca? } \\
\text { *Pregunta cerrada de tipo excluyente }\end{array}$ \\
\hline & $\begin{array}{l}\text { Asignaciones } \\
\text { económicas }\end{array}$ & $\begin{array}{l}\text { ¿Disposición de dinero? } \\
\text { ¿Cantidad de dinero de la que dispone? } \\
\text { ¿Quién da el dinero? } \\
\text { ¿Frecuencia con la que le dan dinero? } \\
\text { ¿El ahorro? } \\
\text { ^Preguntas cerradas de tipo excluyente }\end{array}$ \\
\hline \multirow[t]{2}{*}{$\begin{array}{l}\text { Consumo } \\
\text { responsable }\end{array}$} & $\begin{array}{l}>\text { Actitudes y } \\
\text { comportamiento de } \\
\text { consumo responsable }\end{array}$ & $\begin{array}{l}\text { Actividades relacionadas con el consumo } \\
\text { ¿Comparar precios? } \\
\text { ¿Fijarse en la calidad del producto? } \\
\text { ¿Entiendes el etiquetaje? } \\
\text { ¿Fijarse en la fecha de caducidad? } \\
\text { ¿Guardar la factura? } \\
\text { ¿Pensar en las ventajas e inconvenientes del producto? } \\
\text { ¿Informarse de las características del producto? } \\
\text { ¿Valorar la necesidad de adquirir el producto? } \\
\text { `Pregunta cerrada de tipo ordinal por intensidad } \\
\text { En relación con el consumo } \\
\text { ¿Conoces organismos o entidades a los que te puedes dirigir en } \\
\text { caso de reclamación? } \\
\text { ¿Has tenido educación para un consumo responsable? } \\
\text { ¿Te agradaría aprender más sobre el consumo responsable?. } \\
\text { ¿Consideras que el consumo daña el medio ambiente?' } \\
\text { `Pregunta cerrada de tipo excluyente }\end{array}$ \\
\hline & $\begin{array}{l}>\text { Influencia en el } \\
\text { consumo familiar }\end{array}$ & $\begin{array}{l}\text { ¿Cuán considerada es su opinión al decidir cómo se gasta el } \\
\text { dinero en el hogar? } \\
\text { ¿Tiene importancia su opinión al interior del hogar al comprar } \\
\text { productos? } \\
\text { ¿Decides a qué colegio vas? } \\
\text { ¿Decides el lugar de vacaciones? } \\
\text { ¿Decides lo que se compra en tu hogar? } \\
\text { ^Pregunta cerrada de tipo ordinal por intensidad }\end{array}$ \\
\hline
\end{tabular}

Fuente: elaboración propia considerando las subidmensiones e indicadores para la elaboración del cuestionario 


\subsection{Análisis de la información}

Se realizó un análisis estadístico descriptivo de los datos, se codificaron las respuestas, se creó una matriz y se vaciaron los cuestionarios. Luego se procedió al recuento de respuestas, se elaboraron las gráficas de cada variable de forma individual y se estableció la relación entre las variables. Cabe señalar que se establecen frecuencias, porcentajes, media y desviación típica. Para esto se utilizó el programa estadístico SPSS:19.0.

\section{Resultados}

De las actividades planteadas respecto a la principal afición o hobby, los adolescentes señalan que la actividad que más realizan es salir con amigos, con un 40,0\%. En segundo lugar, la práctica de actividades deportivas, con el 27,0\%. En último lugar, con un 0\%, se encuentra realizar actividades con juegos no electrónicos, artes plásticas (manualidades y pintura) e ir de compras.

En relación con los hábitos de compra, las preguntas: ¿En qué te fijas al comprar un producto?, ¿Aspectos que consideras para comprar un producto? y ¿Motivos en la decisión final de compra?, corresponden a cuestiones de tipo ordinal por intensidad. El objetivo de tales interrogantes es obtener la media para apreciar el rango promedio que le asignan a cada indicador, y la desviación típica para ver el grado de dispersión.

En la pregunta ¿En qué te fijas al comprar un producto? se aprecia que los adolescentes consideran "Bastante", el precio, la utilidad y la necesidad del producto, y se fijan "Poco" en la marca y otros aspectos del producto. Sin embargo, el valor más alto de dispersión se encuentra en el aspecto de que sea un producto concreto, según se muestra en la tabla 5.

Tabla 5. Resultados de aspectos al comprar un producto

\begin{tabular}{|l|c|c|}
\hline $\begin{array}{l}\text { ¿En qué te fijas al comprar un } \\
\text { producto? }\end{array}$ & Media & $\begin{array}{c}\text { Desviación } \\
\text { típ. }\end{array}$ \\
\hline En el precio & 3,60 & 1,15 \\
\hline En el diseño, la estética & 3,09 & 1,27 \\
\hline En que esté de moda & 2,66 & 1,32 \\
\hline $\begin{array}{l}\text { En que sea un producto } \\
\text { concreto (ropa, juegos) }\end{array}$ & 3,21 & 1,38 \\
\hline En la marca & 2,39 & 1,31 \\
\hline En que sea de calidad & 3,21 & 1,30 \\
\hline En la relación calidad / precio & 3,25 & 1,22 \\
\hline En que sea útil o necesario & 3,62 & 1,21 \\
\hline $\begin{array}{l}\text { Otros (todo en general, nada en } \\
\text { concreto, comprar rápido) }\end{array}$ & 1,92 & 1,18 \\
\hline
\end{tabular}

Fuente: elaboración propia a partir de los resultados del cuestionario 
De acuerdo con Aspectos que consideran para comprar un producto, los adolescentes reconocen que "Habitualmente" compran productos que están de moda y que sean de nombres y marcas conocidas. Manifiestan tener "Poco" interés por: comprar productos que han visto en anuncios, tener productos de las mismas marcas que sus amigos, y comprar los mismos productos que compran sus amigos. El mayor grado de dispersión se encuentra en comprar productos de marcas conocidas, con 1,34. Esto se evidencia en la tabla 6.

Tabla 6. Resultados de aspectos para comprar un producto

\begin{tabular}{|l|c|c|}
\hline $\begin{array}{l}\text { Aspectos para comprar un } \\
\text { producto }\end{array}$ & Media & Desviación típica \\
\hline $\begin{array}{l}\text { Acostumbras a comprarte cosas } \\
\text { que están de moda }\end{array}$ & 2,75 & 1,17 \\
\hline $\begin{array}{l}\text { Te gusta comprar productos de } \\
\text { nombres y marcas conocidas }\end{array}$ & 2,62 & 1,34 \\
\hline $\begin{array}{l}\text { Compras productos que has } \\
\text { visto en anuncios }\end{array}$ & 2,09 & 1,10 \\
\hline $\begin{array}{l}\text { Te gusta tener productos de las } \\
\text { mismas marcas que tus amigos }\end{array}$ & 2,06 & 1,13 \\
\hline $\begin{array}{l}\text { Te gusta comprar los mismos } \\
\text { productos que compran tus } \\
\text { amigos }\end{array}$ & 1,98 & 1,08 \\
\hline
\end{tabular}

Fuente: elaboración propia a partir de los resultados del cuestionario

En relación con la frecuencia mensual de compra de productos concretos (ropa, calzado, música, etc.), el 35,3\% señala que esta se realiza una vez al mes; el 31,5\% indica que no sabe cuántas veces realiza esta acción; el 21,1\% afirma que compra dos veces al mes; el $9,1 \%$, tres veces al mes: un 1,7\% reconoce comprar entre cinco y diez veces, mientras que el $0,9 \%$ señala que compra estos productos cuatro veces al mes.

La decisión de compra para los adolescentes está guiada "Habitualmente" por: el precio y la calidad, la cual consideran "Bastante" influyente al la hora de decidir la compra. El mayor grado de dispersión en las respuestas, 1,21, se encuentra precisamente en el precio. Según se muestra en la tabla 7.

Tabla 7. Resultados de la decisión de compra

\begin{tabular}{|l|c|c|}
\hline Decisión de compra & Media & $\begin{array}{c}\text { Desviación } \\
\text { típ. }\end{array}$ \\
\hline Precio & 3,50 & 1,21 \\
\hline Calidad d, & 3,54 & 1,14 \\
\hline Que esté de mModa & 2,58 & 1,14 \\
\hline $\begin{array}{l}\text { Opinión de madres y } \\
\text { padres }\end{array}$ & 2,49 & 1,08 \\
\hline $\begin{array}{l}\text { Opinión de amigos y } \\
\text { amigas }\end{array}$ & 2,44 & 1,18 \\
\hline Opinión del vendedor & 1,55 & 0,96 \\
\hline
\end{tabular}

Fuente: elaboración propia a partir de los resultados del cuestionario 
Respecto a quién los acompaña en la compra, el 32,8\% afirma que los acompaña la madre; un $24,6 \%$ indica que van acompañados por su padre y su madre; el $24,1 \%$ lo hace acompañado por sus amigos/as,; un $8,2 \%$, solos; el 5,2\%, con el padre; un 2,2\%, con su pareja; el $1,7 \%$ con el hermano, y el $0,9 \%$, con otros familiares. Sin embargo, el $44,8 \%$ prefiere que lo acompañen sus amigos, seguido por el 19,0\%, al que le gusta ir con la madre; al $14,7 \%$ le gusta ir con el padre y la madre; un 5\% escoge ir con el padre; el 4,7\% prefiere ir solo; un $4,3 \%$ elige ir con el hermano y el $2,6 \%$ opta por ir acompañado por la pareja, o por otros familiares.

Los adolescentes prefieren que sus amigos los acompañen en sus compras, porque consideran "Bastante" la confianza, sinceridad y comprensión, y pueden comprar lo que quieran. Mientras que les gusta "Poco", debido a: Porque voy más rápido, Porque vamos en coche, Sin ningún motivo especial. No obstante, el mayor grado de dispersión se encuentra en el aspecto de edad (Somos de la misma edad): 1,59. Esto se evidencia en la tabla 8.

Tabla 8. Resultados de quien le gusta que lo acompañe en las compras
\begin{tabular}{|l|c|c|}
\hline $\begin{array}{l}\text { Por qué te gusta que esa persona te } \\
\text { acompañe a comprar }\end{array}$ & Media & Desviación típ. \\
\hline Porque tiene los mismos gustos que yo. & 2,85 & 1,41 \\
\hline Es divertido, lo paso mejor. & 3,47 & 1,44 \\
\hline $\begin{array}{l}\text { Las opiniones y consejos que me da para } \\
\text { escoger y decidir. }\end{array}$ & 3,25 & 1,43 \\
\hline $\begin{array}{l}\text { Por confianza (sinceridad, comprensión, } \\
\text { puedo comprar lo que quiero). }\end{array}$ & 3,63 & 1,27 \\
\hline $\begin{array}{l}\text { Conoce más de moda, está al día, es } \\
\text { más actual y modernoa. }\end{array}$ & 2,48 & 1,43 \\
\hline Porque es quien paga & 2,24 & 1,56 \\
\hline $\begin{array}{l}\text { Mayor libertad (puedo escoger lo que yo } \\
\text { quiero). }\end{array}$ & 3,16 & 1,48 \\
\hline Por la edad (somos de la misma edad). & 2,36 & 1,59 \\
\hline Porque compra más cosas. & 2,06 & 1,35 \\
\hline $\begin{array}{l}\text { Por los consejos (sabe lo que necesito, lo } \\
\text { que vale la pena, me entrega información } \\
\text { que desconozco). }\end{array}$ & 3,11 & 1,36 \\
\hline $\begin{array}{l}\text { Otros (sin ningún motivo especial, porque } \\
\text { voy más rápido, porque vamos en auto). }\end{array}$ & 2,00 & 1,39 \\
\hline \hline
\end{tabular}

Fuente: elaboración propia a partir de los resultados del cuestionario

En relación con la decisión de compra, la mayoría (88,8\%) afirma que acaba decidiendo lo que compra, seguido de un 5,2\%, que señala que acaba decidiendo la madre; un $3,0 \%$ considera que la decisión es del padre y la madre, mientras que un $0,4 \%$ indica que de sus amigos y de otros familiares. 
Respecto a las asignaciones económicas, el 84,5\% de los adolescentes encuestados señala que recibe dinero; el 15,1\% indica que no recibe dinero, y el 0,4\% no responde.

De acuerdo con la cantidad de dinero que recibe semanalmente, el $44,8 \%$ de los jóvenes indica que dispone de entre 5 y 10 euros, y un 2,6\% señala que recibe más de 30 euros a la semana.

En relación con el origen del dinero, el 45,7\% afirma que es entregado por el padre y la madre, el $22,4 \%$ señala que es proporcionado por la madre, un 10,3\% indica que es otorgado por el padre, mientras que el 4,3\% manifiesta que es asignado por sus abuelos y solo un $1,7 \%$ afirma que el dinero es aportado por otros familiares.

El $42,7 \%$ indica que recibe dinero cada semana; un $33,2 \%$ cuando lo pide; el $5,6 \%$, cada día, y un $3,0 \%$, cada mes.

Sobre lo que hacen con el dinero, el 50,4\% indica que ahorra siempre que puede, el $14,2 \%$ afirma que lo distribuye para gastos del mes, el $13,8 \%$ señala que se lo gasta rápidamente, y un 5,2\% considera que no ahorra nunca.

Respecto al gasto de dinero, el 50,9\% señala que gasta el dinero principalmente en salir con sus amigos. Muy por debajo de esta opción se encuentra la segunda preferencia, que consiste en comprar revistas y cómic, con un 6,9\%. La tercera opción, con el 5,6\%: comprar cosas para comer (patatas fritas, chuches). Como cuarta preferencia, con un 4,3\%: comprar ropa, calzado y complementos. En quinto lugar se encuentran juegos de ordenador y consolas, con un 3,4\%. Las actividades en que menos gastan dinero son: tabaco, telefonía móvil, CD de música (2,2\%), ir al cine (1,7\%), libros (1,3\%), mientras que el bar, comida rápida y peluquería solo son considerados por el $0,4 \%$.

En Actitudes y comportamiento de consumo responsable, el 52,2\% de los jóvenes no se considera consumista, mientras que el $45,3 \%$ sí, y un $2,5 \%$ no responde. Así también, el $50,4 \%$ de los adolescentes afirma que compra productos de marcas conocidas, frente a un $46,6 \%$ que señala que no lo hace, y un 3,0\% no responde. Para el $49,6 \%$ de los adolescentes, los productos de marcas conocidas son más caros. Un $25,0 \%$ afirma que son más buenos y más caros, el 13,4\% señala que son más buenos, el $8,2 \%$ indica que no son más buenos ni más caros y un 3,9 escoge la opción "otros".

La pregunta sobre las actividades relacionadas con el consumo, tiene por propósito obtener la media para apreciar el rango promedio que le asignan a cada indicador, y la desviación típica para ver el grado de dispersión. Con base en ello, los adolescentes manifiestan que realizan "Habitualmente" cada una de las actividades mencionadas, aunque 
el mayor grado de dispersión $(1,46)$ se encuentra en el aspecto Guardas la factura. Según se muestra en la tabla 9.

Tabla 9. Resultados de las actividades relacionadas con el consumo

\begin{tabular}{|l|c|c|}
\hline $\begin{array}{l}\text { Actividades relacionadas con el } \\
\text { consumo }\end{array}$ & Media & $\begin{array}{c}\text { Desviación } \\
\text { Típ. }\end{array}$ \\
\hline $\begin{array}{l}\text { Antes de comprar un producto comparas } \\
\text { precios. }\end{array}$ & 3,25 & 1,36 \\
\hline $\begin{array}{l}\text { Te fijas en la calidad del producto antes de } \\
\text { comprar. }\end{array}$ & 3,23 & 1,20 \\
\hline Entiendes el etiquetaje de los productos. & 2,82 & 1,27 \\
\hline $\begin{array}{l}\text { Te fijas en la fecha de caducidad de los } \\
\text { productos. }\end{array}$ & 3,30 & 1,40 \\
\hline $\begin{array}{l}\text { Guardas la factura. } \\
\text { Consideras las ventajas e inconvenientes } \\
\text { (antes de comprar un producto). }\end{array}$ & 3,20 & 1,46 \\
\hline $\begin{array}{l}\text { Valoras la necesidad antes de comprar un } \\
\text { producto. }\end{array}$ & 3,19 & 1,27 \\
\hline
\end{tabular}

Fuente: elaboración propia a partir de los resultados del cuestionario

Respecto a la educación para el consumo, los jóvenes indican que han tenido educación para un consumo responsable, pero les gustaría aprender sobre este tipo de consumo, y afirman que el consumo daña el medio ambiente. Por otro lado, señalan no tener conocimiento de organismos o entidades a los que se pueden dirigir en caso de reclamación. No obstante, el mayor grado de dispersión en las respuestas lo presenta El consumo daña el medio ambiente $(0,63)$. Esto se evidencia en la tabla 10.

Tabla 10. Resultados de educación para el consumo

\begin{tabular}{|l|c|c|}
\hline Educación para el consumo & Media & Desviación típ. \\
\hline $\begin{array}{l}\text { Conoces organismos o } \\
\text { entidades a los que te puedes } \\
\text { dirigir en caso de reclamación. }\end{array}$ & 1,51 & 0,55 \\
\hline $\begin{array}{l}\text { Has tenido educación para un } \\
\text { consumo responsable. }\end{array}$ & 1,05 & 0,34 \\
\hline $\begin{array}{l}\text { Te gustaría aprender sobre el } \\
\text { consumo responsable. }\end{array}$ & 1,43 & 0,55 \\
\hline $\begin{array}{l}\text { Consideras que el consumo } \\
\text { daña el medio ambiente. }\end{array}$ & 1,41 & 0,63 \\
\hline
\end{tabular}

Fuente: elaboración propia a partir de los resultados del cuestionario

La pregunta sobre La influencia en el consumo familiar es de tipo ordinal por intensidad, en la cual se trabajó con cinco categorías que aluden a la escala de Likert. El propósito de esta pregunta es obtener la media para apreciar el rango promedio que le asignan a cada indicador, y la desviación típica para determinar el grado de dispersión. Los 
adolescentes señalan que "Habitualmente" se tiene en cuenta su opinión al comprar productos para el hogar y decidir a qué colegio van. También manifiestan que influyen "Poco" al decidir cómo se gasta el dinero de su hogar, el lugar de vacaciones y lo que se compra en el hogar. El mayor grado de dispersión en las respuestas, con un 1,47, se aprecia en el aspecto Decides a que colegio vas. Según se muestra en la tabla 11.

Tabla 11. Resultados sobre la influencia en el consumo familiar
\begin{tabular}{|l|c|c|}
\hline $\begin{array}{l}\text { Influencia en el consumo } \\
\text { familiar }\end{array}$ & Media & Desviación típ. \\
\hline $\begin{array}{l}\text { Se tiene en cuenta tu opinión al } \\
\text { decidir cómo se gasta el dinero } \\
\text { en tu hogar. }\end{array}$ & 2,29 & 1,20 \\
\hline $\begin{array}{l}\text { Se tiene en cuenta tu opinión al } \\
\text { comprar productos para el } \\
\text { hogar. }\end{array}$ & 2,58 & 1,21 \\
\hline Decides a qué colegio vas. & 3,16 & 1,47 \\
\hline Decides el lugar de vacaciones. & 2,41 & 1,21 \\
\hline $\begin{array}{l}\text { Decides lo que se compra en tu } \\
\text { hogar. }\end{array}$ & 2,13 & 1,02 \\
\hline
\end{tabular}

Fuente: elaboración propia a partir de los resultados del cuestionario

\section{Discusión}

Los resultados despiertan la reflexión de los indicios o tendencias generales derivados del análisis y la implicación suscitada. Respecto a los hábitos de consumo del alumnado de secundaria, en el estudio se evidencia que la principal "afición" de los adolescentes es salir con amigos/as (40,0\%) y realizar actividades deportivas (27,0\%). Estos datos no coinciden con las investigaciones catalanas (Institut Català de Consum, 2004; Querol, Peregrina y Viciana, 2011). El primero indica que las principales tendencias de los adolescentes catalanes son: realizar actividades deportivas (37,3\%) y salir con los amigos (15,3\%). El segundo señala que la principal actividad de los jóvenes catalanes es realizar ejercicios físicos (55,7\%), seguido por las aficiones informáticas (42,1\%). Si bien los datos contrastan, es pertinente considerar que en los tres estudios aparece la actividad física como una de las principales aficiones. Por otro lado, el hecho de que en nuestro estudio la actividad "salir con amigos" sea la principal, pueda deberse a que se realizó en diferentes comarcas, además del área metropolitana. Esto se fundamenta en el hecho de que, durante la adolescencia, las relaciones con amistades cumplen un papel importante, aunque de un modo muy particular, ya que se busca en el grupo de pares un apoyo que refuerce la autonomía respecto al ámbito familiar, de forma progresiva (Carretero, Palacios y Marchesi, 1991). Cabe destacar que en esta etapa de la vida, la elaboración de la identidad personal se realiza mediante la 
interacción, porque durante este periodo, se le asigna un gran valor al grupo de iguales (Palacios, Marchesi y Coll, 1993; Papalia y Wendkos, 1997).

En relación con los hábitos de compra, los adolescentes del estudio manifiestan que al comprar un producto, el precio, la utilidad y la necesidad, suponen factores bastante considerados, mientras la marca y otros aspectos son poco estimados. Asimismo, señalan tener poco interés por: comprar productos que han visto en anuncios y de las mismas marcas que sus amigos. Para ellos, la decisión de compra está guiada habitualmente por el precio y la calidad, la cual afirman que influye bastante al decidir la compra. De los datos señalados, sólo el precio coincide con los resultados del estudio de Fonolleda, Gual y Granados (2013), quienes indican que los criterios más referidos por los jóvenes catalanes al comprar productos de vestir son: el precio $(18,5 \%)$, el gusto $(12,7 \%)$ y la marca $(12,4 \%)$. Mientras, tienen una presencia considerablemente inferior los criterios de calidad $(8,9 \%)$ y diseño (5,2\%). Por otro lado, la investigación de Global Habbo Youth Survey (2009), realizada en 30 países con una muestra de 120000 jóvenes, de los cuales 6000 encuestados eran españoles, contrasta con el dato de nuestra investigación: un 66,0\% declara que casi siempre compra sus marcas favoritas, mientras un $49 \%$ asegura que la marca no afecta a su decisión de compra. Finalmente, el $27 \%$ indica que no le gustan las marcas. Siguiendo con el aspecto de la marca, la investigación Outlook Teens (2010), realizada por Creafutur, en la que participaron adolescentes de 12-19 años, de 8 países (los Estados Unidos, Gran Bretaña, Francia, Brasil, China, Japón, México y España), indica que los adolescentes actuales prefieren los productos de marca, hecho que también contradice los resultados de nuestra investigación. No obstante, se evidencia un matiz importante: en los países emergentes (China con un $75 \%$, Brasil con un $69 \%$ y México con un $61 \%$ ), los adolescentes afirman comprar siempre productos de marcas conocidas. Los franceses y españoles son quienes menos marcas consumen, con un 45\% y un 33\%, respectivamente. Este dato es considerable, porque refleja que los adolescentes españoles están preocupados por su imagen al escoger las marcas, aunque en menor escala que en otros países.

En cuanto a las Asignaciones económicas, un estudio reciente realizado por Blasco y Pintado (2014), indica que los jóvenes españoles entre 12-19 años, reciben una paga media de 13,5 euros semanales, aunque consideran que sus padres deberían asignarles una paga que rondara los 16,12 euros semanales. Del dinero recibido solo destinan al ahorro 1,3 euros de media. Otro dato interesante es que el $80 \%$ de los adolescentes recibe algún dinero extra 
a la semana, independientemente de si reciben paga o no. Reconocen gastar una media de 12,2 euros a la semana, pero su gasto se acerca a los 38,46 euros por semana. En este mismo ámbito se encuentra el estudio de Querol et al. (2011), en el que indican que aproximadamente el $20 \%$ de los jóvenes catalanes dispone de menos de 80 euros al mes, y la media que reciben los adolescentes entre 12-16 años es de 31,13 euros mensuales. Más del $70 \%$ de los alumnos entre 12-18 años, afirma recibir dinero cada vez que lo pide, y la mayoría de los jóvenes gasta el dinero en ocio, principalmente: ir al cine y salir con amigos. Los datos aportados por las investigaciones citadas coinciden con los resultados de nuestro estudio, ya que el $44,8 \%$ de los jóvenes indica que la cantidad de dinero de la cual dispone semanalmente, oscila entre 5 y 10 euros, y sólo un 2,6\% señala que recibe más de 30 euros a la semana. Respecto a la frecuencia con la que recibe dinero, el $42,7 \%$ indica recibirlo cada semana, un $33,2 \%$ sólo cuando lo pide, el 5,6\% cada día y un 3,0\% cada mes. En relación con lo que hacen con el dinero, el 50,4\% indica que ahorra siempre que puede, el $14,2 \%$ afirma que lo distribuye para gastos del mes, el 13,8\% señala que se lo gasta rápidamente, y sólo un 5,2\% considera que no ahorra nunca. Es destacable el interés mostrado por el ahorro, lo que denota la existencia de este hábito entre ellos. Gastan el dinero principalmente en salir con sus amigos, lo que indica la importancia que brindan a relacionarse con sus pares en esta etapa de la vida (Palacios et al., 1993; Papalia y Wendkos, 1997).

En relación con el Consumo responsable y las Actitudes y comportamiento de este tipo de consumo, se debe tener en cuenta que la mayoría $(52,2 \%)$ de los adolescentes de nuestro estudio no se consideran consumistas y el 50,4\% declara que le gusta comprar productos de marcas conocidas. Esto se corrobora con los datos del estudio Institut Català de Consum (2004), donde se indica que al 62,0\% de los adolescentes catalanes le gusta comprar productos de marcas y nombres conocidos. Sin embargo, esta cifra baja considerablemente en el estudio Fonolleda, et al. (2013), ya que un $12,4 \%$ de los adolescentes manifiesta que se fija bastante en la marca al comprar ropa. Este factor puede ser el que produce la baja significativa, debido a que en los estudios anteriores se preguntaba por productos en general, y en el último se cuestiona de forma de específica por la marca en la compra de ropa.

Más allá de lo que se pueda pensar, las respuestas de los adolescentes dejan en evidencia que se fijan "Bastante", o de forma "Habitual", antes de comprar un producto, en los siguientes aspectos: comparar precios, calidad, entender el etiquetaje, mirar la fecha de 
caducidad, guardar la factura, considerar las ventajas e inconvenientes y valorar la necesidad de la compra. Estos datos coinciden con los del Institut Català de Consum (2004).

Respecto a la Educación para el consumo, los jóvenes de Barcelona consideran que han tenido suficiente educación para un consumo responsable, pero lo interesante es que indican que les gustaría aprender más sobre este tipo de consumo. Sin embargo, señalan no tener conocimiento de organismos o entidades a los que se pueden dirigir en caso de reclamación. Esto último se corresponde con el estudio de Tubau, Alonso y Gómez (2011), quienes indican que el $80 \%$ de los adolescentes catalanes solo conoce uno o dos derechos del consumidor, y que el porcentaje de quienes conocen dos o tres derechos, es muy parecido al de jóvenes que no conocen ni un derecho del consumidor, próximo al $20 \%$.

Sobre La influencia en el consumo familiar, manifiestan que habitualmente se toma en consideración, en el entorno familiar, su opinión al comprar productos para el hogar y decidir a qué colegio van, pero esta influencia es poca al : decidir el lugar de vacaciones, lo que se gasta, y lo que se compra en el hogar. Estos antecedentes coindicen con la investigación de Outlook Teens (2010), que señala que los jóvenes tienen una gran influencia en las decisiones de compra familiar, y 6 de cada 10 participan activamente en la compra de alimentos y productos del hogar.

\section{Conclusiones}

Se ha reconstruido la realidad a través de la descripción de un fenómeno. Se conoce que estos datos pueden ser el inicio para la configuración de nuevas investigaciones. Más allá de esto, es importante destacar que los adolescentes han manifestado que sus hábitos de consumo son moderados. En ellos no se percibe un consumo exagerado, en absoluto consumista. Sin embargo, diversas investigaciones sobre el tema indican que los adolescentes de hoy tienen hábitos y valores consumistas y que en ello ha contribuido la sociedad de consumo, aunque aluden a la familia como la gran responsable en el plano educativo, siendo esta el contexto en el que se inician. Ante tal dicotomía, consideramos que es difícil saber si los encuestados responden con base en sus verdaderas necesidades e intereses, o simplemente desde la perspectiva del hábito social. Esto se fundamenta en la necesidad de las personas por obtener aprobación social de los otros, respondiendo de un modo culturalmente aceptable y apropiado, pero se trata de un riesgo que se corre en todas las investigaciones, cuando se trabaja con la opinión de las personas. 
A pesar de lo expuesto, los antecedentes recopilados en el estudio apuntan a la necesidad de realizar una propuesta pedagógica para la educación del consumo desde el ámbito de los valores. Esto se basa en la directa relación que existe entre éstos, en tanto constituyen parte de una educación integral. Si el objetivo es formar personas íntegras, con capacidad crítica, reflexiva y autónoma, la simple transmisión de conocimientos de manera parcelada, no sirve. La educación para el consumo se debe realizar para que los ciudadanos tomen conciencia de su condición de personas consumidoras, entendiendo este tipo de educación como una oportunidad para ayudar a los individuos a construir su modo de situarse frente al mundo, siendo la adolescencia una etapa clave para ello (Fonolleda et al., 2013).

Por lo tanto, se sugiere elaborar propuestas de programas educativos en el área, con el fin de desarrollar talleres que contengan actividades de educación para el consumo. La realización de un programa se basa en la necesidad de tener una guía de trabajo sobre el tema, a través de todas las asignaturas, o de una específica. Respecto a la implementación de actividades, se propone que sean didácticas y utilicen los medios audiovisuales. Todo esto debe apoyarse en el trabajo cooperativo de la comunidad educativa, y se sustenta en la necesidad de involucrar a las familias y a los docentes. Como ya se vislumbró, existe un trinomio Alumno-Familia-Instituto, y es aquí dónde se debe trabajar la educación para el consumo, porque educar para el consumo en el siglo XXI, significa que tenemos el reto de formar ciudadanos activos, responsables y críticos.

\section{Referencias}

Álvarez, Luis y Álvarez, Nieves. (1988). El consumo va a la escuela. Barcelona: Laia.

Arnould, Eric y Thompson, Craig. (2005). Consumer culture theory (CCT): Twenty years of Research. Journal of Consumer Research, 31(4), 868-882.

Autio, Minna. (2002). Finnish young people's narrative construction of consumer identity. International. Journal of Consumer Studies, 28(4), 388-398.

Baker, Andrew, Moschis, George, Benmoyal-Bouzaglo, Sara y Pizzutti dos Santos, Cristiane. (2013). How Family Resources Affect Materialism and Compulsive Buying: A CrossCountry Life Course Perspective. Cross-Cutural Research, 47(4), 335-362.

Belch, Michael, Krentler, Kathleen y Willis-Flurry, Laura. (2005). Teen internet mavens: Influence in family decision making. Journal of Business Research, 58(6), 569-575.

Benn, Jette. (2002). Consumer education: educational considerations and perspectives. International Journal of Consumer Studies, 26(3), 169-177. 
Blasco, Francis y Pintado, Teresa. (2014). Adolescentes 2013: hacia una correcta formación y autonomía en el consumo. Pamplona: Círculo Rojo.

Burroughs, James y Rindfleisch, Aric. (2002). Materialism and well-being: A conflicting values perspective. Journal of Consumer Research, 29(3), 348-370.

Buxarrais, María Rosa. (2003). Los valores en una sociedad individualista y consumista. Revista de la Confederación Estatal de Asociaciones de Padres y Madres de Alumnos. Madrid: España, (76), 7-9.

Cantera, Desiderio. (2002). Educación para el consumo. Recuperado de http://www.redescepalcala.org/inspector/DOCUMENTOS\%20Y\%20LIBROS/ESCUELA\%20DE\%20PAD RES/CEAPA/EDUCACION\%20PARA\%20EL\%20CONSUMO.htm

Carioso, Alba. (2008). El consumo en la encrucijada ética. Revista Utopía y Praxis Latinoamericana, 13(41), 13-45.

Carretero, Mario, Palacios, Jesús y Marchesi, Álvaro. (1991). Psicología evolutiva: Adolescencia, madurez y senectud. Madrid: Alianza.

Cohen, Joel y Bernard, Russell. (2013). Evolutionary psychology and consumer behavior: A constructive critique. Journal of Consumer Psychology, 23(3), 387-399.

Cortina, Adela. (2002). Por una ética del consumo. Madrid: Taurus.

Crane, Daniel. (2010). Cambio en el medio ambiente y el futuro del consumo: implicaciones para la identidad del consumidor. Anuario Filosófico, 43(2), 353-379.

Chaplin, Lan Nguyen y John, Deborah. (2010). Interpersonal influences on adolescent materialism: A new look at the role of parents and peers. Journal of Consumer Psychology, 20, 176-184.

Chaplin, Lan Nguyen y Lowrey, Tina. (2010). The Development of Consumer-Based Consumption Constellations in Children. Journal of Consumer Research. 36(5), 757777.

España, Ministerio de Sanidad y Consumo. (2004). Educación y protección del consumidor. España: El Ministerio.

España, Creafutur. (2010). Outlook Teens 2010. Com són els adolescents d'avui i com evolucionaran els seus hàbits de consum. Barcelona, España: Generalitat de Catalunya/ ESADE. Recuperado de http://www.esade.edu/itemsweb/content/produccion/4002241.pdf

Ersoy-Quadir, Seher. (2012). An Analysis of Some of the Factors Behind Materialism Among University Students in Turkey. Journal of adult development, 19(2), 79-87.

Fernández Cavia, José. (2000). El consumidor adolescent. Motivaciones, actitudes $i$ comportament dels adolescents devant el consum, les marques i la publicitat. (Tesis doctoral). Universitat Autònoma de Barcelona, Barcelona, España. 
Flouri, Eirini. (1999). An integrated model of consumer materialism: Can economic socialization and maternal values predict materialistic attitudes in adolescents? Journal of Socio-Economics, 28(6), 707-724.

Flouri, Eirini. (2004). Exploring the relationship between mothers' and fathers' parenting practices and children's materialist values. Journal of Economic Psychology, 25(6), 743-752.

Fonolleda, Marta, Gual, Marta y Granados, Sílvia. (2013). Els joves el consum de roba $i$ complements. Estudis sobre els hàbits de consum dels joves de Catalunya. Barcelona: Generalitat de Catalunya, Escola del Consum de Catalunya y Agència Catalana del Consum.

Fromm, Eric. (1976). Del tener al ser. Barcelona: Paidós.

García Canclini, Néstor. (1995). Consumidores y ciudadanos: conflictos multiculturales de la globalización. México DF: Grijalbo.

Global Habbo Youth Survey. (2009). Uncovers American Teens' Unique Attitudes Towards Brands. Recuperado de http://www.sulake.com/press/releases/habbo-global-youthsurvey-2009-reveals-the-brands-at-the-forefront-of-teens-minds/

Goldsmith, Ronald y Clark, Ronald. (2012). Materialism, Status Consumption, and Consumer Independence. Journal of Social Psychology, 152(1), 43-60.

Griskevicius, Vladas y Kenrick, Douglas. (2013). Fundamental motives: How evolutionary needs influence consumer behavior. Journal of Consumer Psychology, 23(3), 372-386.

Hernández, Roberto, Fernández, Carlos y Baptista, Pilar. (2003). Metodología de la investigación. (3a. ed.). México: Mc Graw Hill.

Hill, William y Beatty, Sharon. (2011). A model of adolescents' online consumer self - efficacy (OCSE). Journal of Business Research, 64, 1025-1033.

Institut Catalá del Consum. (2004). El consum responsable. Estudi de les actituds $i$ el comportament dels adolescents catalans en relació al consum. Barcelona: Generalitat de Catalunya.

Isakesen, Katja y Roper, Stuart. (2008). The impact of Brading on Low Income Adolescents Avisious Cycle? Psychology \& Marketin, 25(11), 1063-1087.

Kapferer, Jean-Nöel. (1992). La marca, capital de la empresa. Bilbao: Deusto.

Lazo, Carmen y Gabelas, José Antonio. (2007). La educación para el consumo de pantallas como praxis holística. Revista Latina de Comunicación Social, 10(62), 1-5.

Mateo, Joan. (2001). La investigación educativa. Dossier del Programa de doctorado: Calidad Educativa en un Mundo Plural. Departamento de métodos de investigación y diagnóstico en educación. Barcelona: Universidad de Barcelona. 
Martín, Manuel y Velarde, Olivia. (2000). Informe Juventud en España, 2000. Madrid: Instituto de la Juventud, Ministerio de Trabajo y Asuntos Sociales.

Osuna, Sara. (2008). Publicidad y consumo en la adolescencia. La educación de la ciudadanía. Barcelona: Icaria Antrazyt.

Palacios, Jesús, Marchesi, Álvaro y Coll, César. (1993). Desarrollo psicológico y educación. Madrid: Alianza.

Papalia, Diane y Wendkos, Sally. (1997). Psicología del desarrollo. Séptima edición. Colombia: McGraw-Hill

Park, Ji Kyung y John, Deborah. (2011). More than meets the eye The influence of implicit and explicit esteem materialism. Journal Consumer Psychology, 21(1), 73-87.

Pham, Michael. (2013). The seven sins of consumer psychology. Journal of consumer psychology, 23(4), 411-423.

Posadas, Ruslan. (2013). La vida de consumo o la vida social que se consume: apreciaciones sobre la tipología ideal del consumismo de Zygmunt Bauman. Estud. Polít (Méx), (29), 115-127.

Pujol, Rosa María. (1996). Educación y consumo. La formación del consumidor en la escuela. Barcelona: ICE-Horsori.

Querol, María, Peregrina, Yessica y Viciana Salvador. (2011). Els joves i els diners. Estudis sobre els hàbits de consum dels joves de Catalunya. Barcelona: Generalitat de Catalunya, Escola del Consum de Catalunya y Agència Catalana del Consum

Roberts, James, Manolis, Cris y Tanner Jeff. (2006). Adolescent autonomy and the impact of family structure on materialism and compulsive buying. Journal of Marketing Theory and Practice, 14(4), 301-314.

Roberts, James, Manolis, Chris y Tanner, Jeff. (2008). Interpersonal influence and adolescent materialism and compulsive buying. Social Influence, 3(2), 114-131.

Saldaña, Dulce Eloísa y Ballesteros, Carlos. (2011). La familia, la cultura y la toma de decisiones en el consumo: Una contribución al comportamiento del consumidor: Madrid: Editorial Académica Española.

Sánchez, Lorenzo, Megías, Ignacio y Rodríguez, Elena. (2004). Jóvenes y publicidad: valores en la comunicación publicitaria para jóvenes. Madrid: INJUVE-FAD.

Shavitt, Sharon, Torelli, Carlos y Wong, Jimmy. (2009). Identity-based motivation, constraints, and opportunities in consumer research. Journal of Consumer Psychology, 19(3), 261266.

Sener, Arzu. (2011). Influences of adolescents on family purchasing behavior: Perceptions of adolescents and parents. Social Behavior and Personality, 39(6), 747-754. 
Shim, Soyeon, Serido, Joyce y Barber, Bonnie. (2011). A Consumer Way of Thinking Linking Consumer Socialization and Consumption Motivation Perspectives to Adolescent Development. Journal of Research on Adolescence, 21(1), 290-299.

Sweeting, Helen, Hunt, Kate y Bhaskar, Abita . (2012). Consumerism and well-being in early adolescence. Journal of Youth Studies, 15(6), 802-820.

Tubau, Toni, Alonso, Elisenda y Gómez, Ruth. (2011). Els joves i el coneixement dels drets $i$ els deures de les persones consumidores. Estudis sobre els hàbits de consum dels joves de Catalunya. Barcelona: Generalitat de Catalunya, Escola del Consum de Catalunya y Agència Catalana del Consum

Wang, Sijun, Holloway, Bets, Beatty, Sharon, y Hill, William. (2007). Adolescent influence in family purchase decisions: An update and cross-national extension. Journal of Business Research, 60(11), 1117-1124. 